\title{
Potassium Channel Distribution, Clustering, and Function in Remyelinating Rat Axons
}

\author{
Matthew N. Rasband, ${ }^{1}$ James S. Trimmer, ${ }^{3}$ Thomas L. Schwarz, ${ }^{4}$ S. Rock Levinson, ${ }^{5}$ Mark H. Ellisman, ${ }^{6}$ \\ Melitta Schachner, ${ }^{7}$ and Peter Shrager ${ }^{2}$ \\ Departments of ${ }^{1}$ Biochemistry and Biophysics and ${ }^{2}$ Neurobiology and Anatomy, University of Rochester Medical Center, \\ Rochester, New York 14642, 'Department of Biochemistry and Cell Biology, State University of New York, Stony Brook, \\ New York 11794, ${ }^{4}$ Department of Molecular and Cellular Physiology, Beckman Center, Stanford University, Stanford, \\ California 94305, 5Health Sciences Center, University of Colorado, Denver, Colorado 80262, ${ }^{\circ D e p a r t m e n t ~ o f ~}$ \\ Neurosciences, University of California San Diego, La Jolla, California 92093-0608, and 7Zentrum fur Moleculare \\ Neurobiologie, Universitat Hamburg, Hamburg, Germany D-20246
}

The $\mathrm{K}^{+}$channel $\alpha$-subunits $\mathrm{Kv} 1.1$ and $\mathrm{Kv1} 1.2$ and the cytoplasmic $\beta$-subunit $\mathrm{Kv} \beta 2$ were detected by immunofluorescence microscopy and found to be colocalized at juxtaparanodes in normal adult rat sciatic nerve. After demyelination by intraneural injection of lysolecithin, and during remyelination, the subcel-

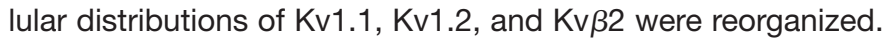
At $6 \mathrm{~d}$ postinjection (dpi), axons were stripped of myelin, and $\mathrm{K}^{+}$channels were found to be dispersed across zones that extended into both nodal and internodal regions; a few days later they were undetectable. By $10 \mathrm{dpi}$, remyelination was underway, but Kv1.1 immunoreactivity was absent at newly forming nodes of Ranvier. By $14 \mathrm{dpi}, \mathrm{K}^{+}$channels were detected but were in the nodal gap between Schwann cells. By 19 dpi, most new nodes had Kv1.1, Kv1.2, and Kv $\beta 2$, which precisely colocalized. However, this nodal distribution was transient. By $24 \mathrm{dpi}$, the majority of $\mathrm{K}^{+}$channels was clustered within paranodal regions of remyelinated axons, leaving a gap that overlapped with $\mathrm{Na}^{+}$channel immunoreactivity. Inhibition of Schwann cell proliferation delayed both remyelination and the development of the $\mathrm{K}^{+}$channel distributions described. Conduction studies indicate that neither 4-aminopyridine (4AP) nor tetraethylammonium alters normal nerve conduction. However, during remyelination, 4-AP profoundly increased both compound action potential amplitude and duration. The level of this effect matched closely the nodal presence of these voltagedependent $\mathrm{K}^{+}$channels. Our results suggest that $\mathrm{K}^{+}$channels may have a significant effect on conduction during remyelination and that Schwann cells are important in $\mathrm{K}^{+}$channel redistribution and clustering.

Key words: potassium channels; demyelination; remyelination; Schwann cells; axons; node of Ranvier
The rapid conduction velocity and successful transmission of electric signals in mammalian myelinated axons depend on the proper spatial distribution of voltage-gated ion-selective channels. $\mathrm{Na}^{+}$channels are clustered at nodes of Ranvier in densities that are $\sim 25$ times that of internodal regions (Shrager, 1989). These clusters are necessary for inward $\mathrm{Na}^{+}$currents at nodes and permit rapid saltatory conduction. In contrast, studies have shown that $\mathrm{K}^{+}$currents appear only after loosening of the myelin sheath from the axonal membrane (Chiu and Ritchie, 1980). This result has been interpreted to imply that $\mathrm{K}^{+}$channels are found only in paranodal and internodal regions and that they normally do not contribute to the action potential. Recent immunocytochemical evidence has confirmed that voltage-gated $\mathrm{K}^{+}$channels are not present at the node in mammalian myelinated nerve. Specifically, two members of the Shaker gene subfamily, Kv1.1 and Kv1.2, have been shown to be present at juxtaparanodal regions adjacent

\footnotetext{
Received Sept. 17, 1997; accepted Oct. 9, 1997.

This work was supported by Grant RG2687 to P.S. from the National Multiple Sclerosis Society and National Institutes of Health Grants NS17965 to P.S.; NS34383 to J.S.T.; GM42376 to T.L.S.; NS15879 to S.R.L.; and RR04050, NS14718, and NS26739 to M.H.E. We thank Ellen Brunschweiger and Ian Vabnick for excellent technical assistance.

Correspondence should be addressed to Dr. Peter Shrager, Department of Neurobiology and Anatomy, Box 603, University of Rochester Medical Center, 601 Elmwood Avenue, Rochester, NY 14642.

Copyright (C) 1997 Society for Neuroscience $\quad 0270-6474 / 97 / 180036-12 \$ 05.00 / 0$
}

to the node of Ranvier, probably as heteromultimers (Wang et al., 1993; Mi et al., 1995).

Disruption or removal of the myelin sheath by disease or injury results in conduction block caused by both increased membrane capacitance and decreased membrane resistance. Demyelination can be repaired in the peripheral nervous system by Schwann cell elaboration of new myelin. However, there are several differences in remyelinated nerve fiber architecture, as compared with axons before injury or disease. The number of myelin lamellae is decreased, and the number of nodes per unit length increases. Regions that were formerly internodal, with low ion channel densities, may have new nodes of Ranvier with high densities of ion channels necessary for saltatory conduction (Ritchie et al., 1981).

Juxtaparanodal $\mathrm{K}^{+}$channels normally are isolated electrically but are uncovered during demyelination, reducing excitability. It has been shown that pharmacological compounds known to block voltage-dependent $\mathrm{K}^{+}$channels can improve conduction in demyelinated axons (Bostock et al., 1981). However, events in remyelination may sequester paranodal $\mathrm{K}^{+}$channels and render them resistant to drugs. Although $\mathrm{Na}^{+}$channel distributions subsequent to demyelination have been well characterized both electrophysiologically (Bostock and Sears, 1978; Shrager, 1987) and immunocytochemically (Dugandzija-Novakovic et al., 1995; Novakovic et al., 1996), much less is known about $\mathrm{K}^{+}$channels. 

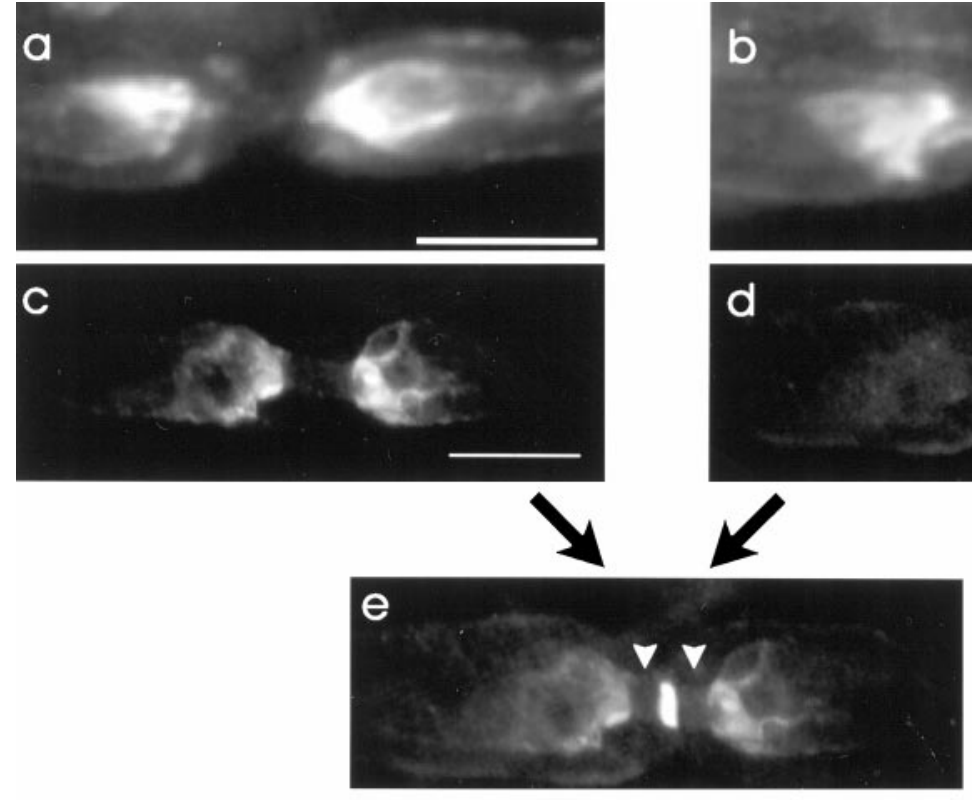
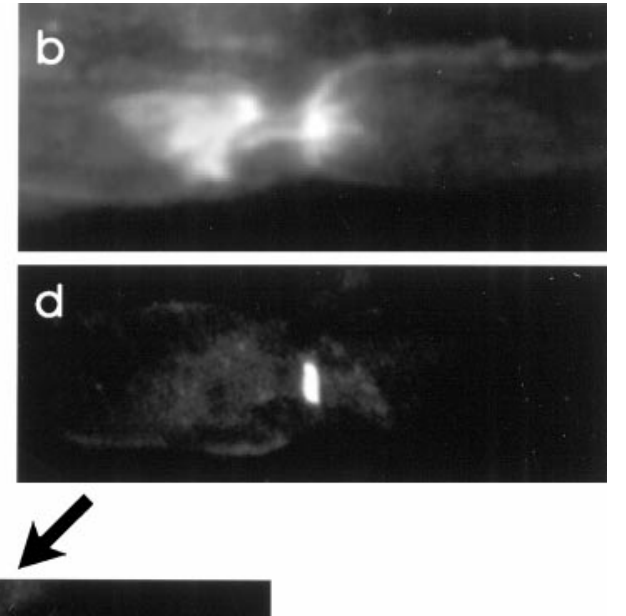
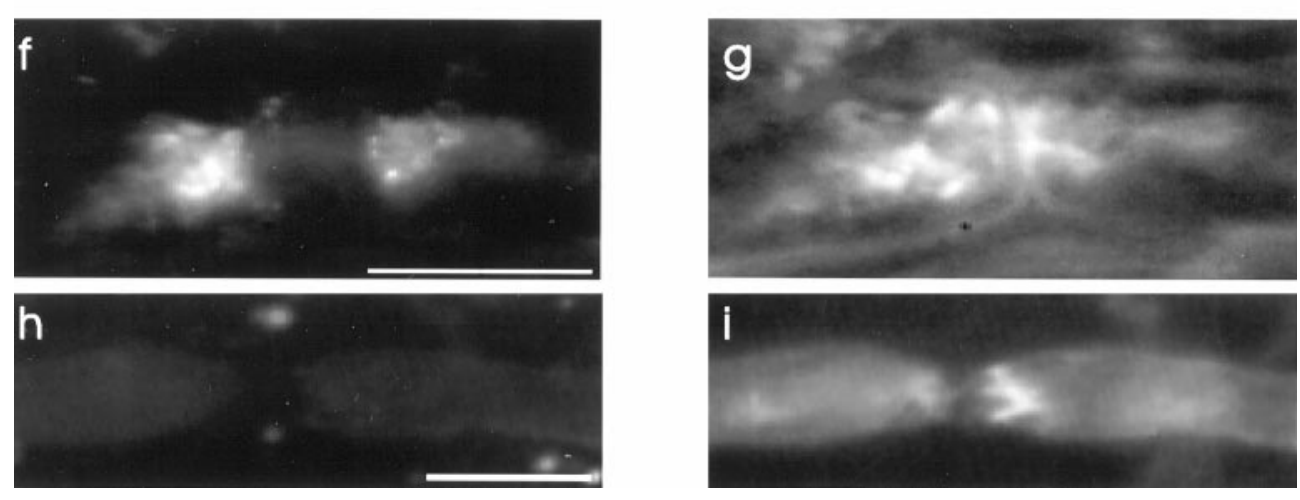

Figure 1. The distribution of Kv1.1 channels in normal adult rat sciatic nerve. $a, b$, A node double-labeled for $\operatorname{Kv1.1}(a)$ and MAG $(b)$. The paranodal localization of MAG indicates that Kv1.1 is mainly just outside of this zone. $c, d, \mathrm{~A}$ node double-labeled for Kv1.1 (c) and $\mathrm{Na}^{+}$channels $(d)$. $e$, A merged image of $c$ and $d$ illustrates the gaps (arrowheads) between $\mathrm{Kv} 1.1$ and $\mathrm{Na}^{+}$channel immunoreactivity. $f, g$, A cryosectioned node double-labeled for Kv1.1 $(f)$ and MAG $(g) . h, i$, A control node labeled with preabsorbed rabbit anti-Kv1.1 $(h)$ and anti-MAG $(i)$. Scale bars, $25 \mu \mathrm{m}$.
Some electrophysiological studies have examined $\mathrm{K}^{+}$currents after demyelination (Ritchie et al., 1981) and during nerve regeneration (Kocsis et al., 1982; Ritchie, 1982). However, to our knowledge, a precise description of the distribution of voltagedependent $\mathrm{K}^{+}$channels during remyelination and their relation to both electrophysiological recordings and neuronal function has not been performed. We describe here the events surrounding redistribution and clustering of the $\mathrm{K}^{+}$channels Kv1.1 and Kv1.2, the involvement of Schwann cells in this redistribution, and the functional consequences of these changes.

Portions of this work have appeared in abstract form (Rasband et al., 1996).

\section{MATERIALS AND METHODS}

Demyelination. Adult female Lewis rats were anesthetized, and the sciatic nerve in one leg, just proximal to the sciatic notch, was exposed. The three branches of the sciatic nerve were injected with 1-2 $\mu \mathrm{l}$ of $1 \%$ lysolecithin in sterile Locke's solution by using a glass micropipette broken to a tip diameter of $\sim 20 \mu \mathrm{m}$. Then the incision was closed, and the animal was allowed to recover for 6-69 d. Some animals were injected with both lysolecithin and mitomycin-C (400 mg/ml; Sigma, St. Louis, MO) to block Schwann cell proliferation subsequent to demyelination. The Locke's solution contained (in $\mathrm{mm}$ ): $\mathrm{NaCl} 154, \mathrm{KCl}$ 5.6, $\mathrm{CaCl}_{2}$ 2, and HEPES 10, $\mathrm{pH} 7.4$.

Axon preparation. On the appropriate day postinjection, the animal was killed; the sciatic nerve was dissected immediately, desheathed, and dissociated into single fibers by treatment with collagenase $(3.6 \mathrm{mg} / \mathrm{ml}$; Sigma) for $1 \mathrm{hr}$ at room temperature (RT). Fibers were teased apart gently and spread on coverslips that had been coated in a few spots with Cell-Tak (Collaborative Research, Bedford, MA). After teasing, the preparations were fixed in $4 \%$ paraformaldehyde in $0.1 \mathrm{M}$ phosphate buffer (PB), pH 7.2, for $30 \mathrm{~min}$, rinsed in $0.05 \mathrm{M} \mathrm{PB}, \mathrm{pH}$ 7.4, for $10 \mathrm{~min}$, and air-dried. Alternatively, for cryosectioned preparations, rats were anesthetized and perfused with $4 \%$ paraformaldehyde in $0.1 \mathrm{M} \mathrm{PB}$. The sciatic nerve was dissected and post-fixed in $4 \%$ paraformaldehyde for 4 $\mathrm{hr}$, rinsed in $0.1 \mathrm{M}$ PB for $10 \mathrm{~min}$, incubated overnight in $20 \%$ sucrose, and incubated again in $30 \%$ sucrose overnight. Then the nerve was frozen in OCT mounting medium (Miller) and cut in 30- $\mu \mathrm{m}$-thick sections. These sections were spread on gelatin-coated slides, fixed again for $10 \mathrm{~min}$ in $4 \%$ paraformaldehyde, rinsed in $0.05 \mathrm{M} \mathrm{PB}$, and allowed to dry.

Immunofluorescence. Tissue preparations were permeabilized for $2 \mathrm{hr}$ in $0.1 \mathrm{M} \mathrm{PB}, \mathrm{pH} 7.4$, containing $0.3 \%$ Triton $\mathrm{X}-100$ and $10 \%$ goat serum (PBTGS). In all steps involving antibodies, the tissue preparations were washed three times for 5 min each with PBTGS between succeeding steps. All antibodies were diluted in PBTGS. The tissue was incubated with the primary antibody overnight. After being washed, the secondary antibody, a goat anti-rabbit IgG conjugated to biotin (1:800, Sigma), was incubated with the tissue. In some experiments, goat anti-rabbit Fcspecific $\mathrm{Fab}_{2}$ fragments conjugated to biotin were used as the secondary antibody (1:400; Accurate Chemicals, Westbury, NY). Then the tissue was incubated with ExtrAvidin-FITC (1:200; Sigma). Double-labeling was performed, using mouse monoclonal antibodies. These were incubated with goat anti-mouse antibody conjugated with TRITC (Sigma). The preparations were air-dried and mounted on slides with an anti-fade mounting medium. In control experiments, primary anti-Kv1.1 antibod- 

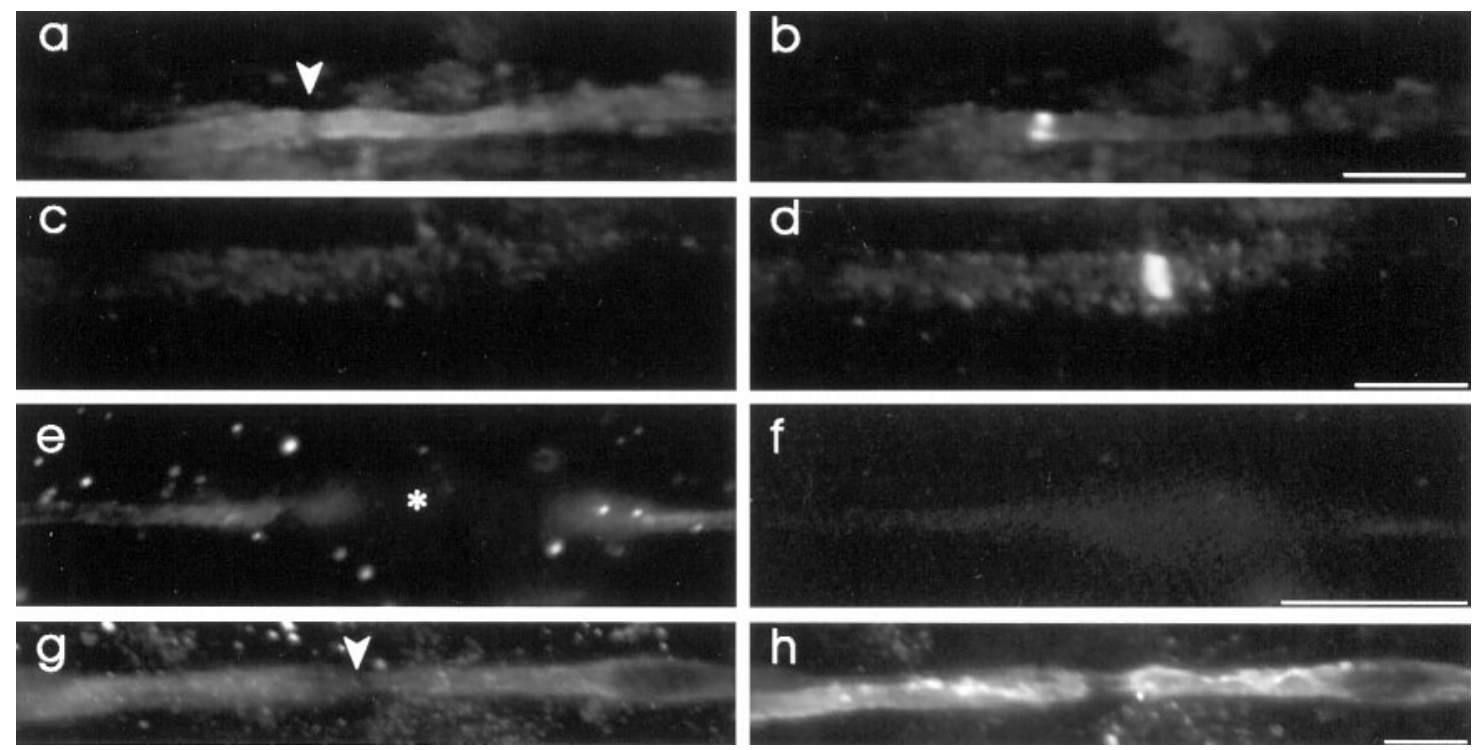

Figure 2. Kv1.1 distributions in demyelinated and early remyelinating axons. $a, b$, A node, at 6 dpi, double-labeled for Kv1.1 ( $a)$ and Na ${ }^{+}$channels $(b)$ has lost the restriction of Kv1.1 to the juxtaparanode. Instead, the Kv1.1 immunoreactivity has moved into nodal (arrowhead) and paranodal zones, whereas $\mathrm{Na}^{+}$channels remain very focal. $c, d$, A node, at 6 dpi, double-labeled for Kv1.1 $(c)$ and $\mathrm{Na}^{+}$channels $(d)$. Kv1.1 staining is undetectable in many demyelinated axons at this time. $e, f$, A demyelinated axon, at $7 \mathrm{dpi}$, double-labeled for Kv1.1 (e) and MAG $(f)$. In $e$, weak Kv1.1 staining was observed adjacent to an associated cell $(*) . g, h$, A new presumptive node, at 9 dpi, double-labeled for Kv1.1 $(g)$ and MAG $(h)$. Remyelinating Schwann cells had diff use cytoplasmic Kv1.1 staining, but no immunoreactivity was detected at the presumptive node (arrowhead). Diff use MAG immunoreactivity indicates early remyelination. Scale bars: $a, b, g, h, 25 \mu \mathrm{m} ; c, d, e, f, 12 \mu \mathrm{m}$.

ies were preincubated in excess peptide antigen before their use for labeling.

The labeled tissue was examined on a Nikon Microphot fluorescence microscope fitted with an SIT 68 camera (Dage-MTI, Michigan City, IN). The camera was connected to a DSP 2000 image processor (DageMTI), which passed images to a DT3851 frame processor in a laboratory computer. Distances were measured by a pair of digital linear gauges with an accuracy of $\pm 1 \mu \mathrm{m}$ (EG-255, Ono Sokki Technology, Osaka, Japan), mounted directly to the microscope stage. Coordinates and distances were relayed to and stored in the computer.

Primary antibodies. For Kv1.1 localization, rabbit polyclonal antibodies were raised against the C-terminal sequence EDMNNSIAHYRQANIRTG (amino acids 458-475). Peptides were synthesized at the Beckman Center, Stanford University (Stanford, CA), and were coupled to porcine thyroglobulin with glutaraldehyde. Immunization, serum collection, and purification were as described in Mi et al. (1995). For immunofluorescence, the polyclonal rabbit anti-Kv1.1 antibodies were diluted 1:30 in PBTGS. For $\mathrm{Na}^{+}$channel immunolocalization, rabbit polyclonal antibodies were raised against a highly conserved 18 -amino-acid peptide (TEEQKK YYNAMKKLGSKK) located between domains III and IV in the vertebrate $\mathrm{Na}^{+}$channel $\alpha$-subunit. The peptide was synthesized at the University of Colorado Medical School institutional facility (Denver, $\mathrm{CO}$ ) and was conjugated to maleimide-activated keyhole-limpet hemocyanin $(\mathrm{KLH})$. Anti-Na ${ }^{+}$channel antibodies were purified by affinity chromatography (ImmunoPure $\mathrm{Ag} / \mathrm{Ab}$ Kit 2, Pierce, Rockford, IL) and used at a dilution of 1:50. Anti-myelin-associated glycoprotein (antiMAG) monoclonal antibodies were prepared as described in Poltorak et al. (1987). The mouse monoclonal anti-Kv1.1 antibody K20/78 (BekeleArcuri et al., 1996) was produced from a mouse immunized with a synthetic peptide (CEEDMNNSIAHYRQANIRTG) corresponding to amino acids $458-476$ of rat Kv1.1. The mouse monoclonal anti-Kv1.2 antibody K14/16 (Bekele-Arcuri et al., 1996; Shi et al., 1996) was produced from a mouse immunized with a glutathione $S$-transferase (GST) fusion protein containing amino acids $428-499$ of rat Kv1.2. K14/16 subsequently was found to bind to a synthetic peptide corresponding to amino acids $463-480$ of Kv1.2. The mouse monoclonal anti-Kv $\beta 2$ antibody K17/70 (Bekele-Arcuri et al., 1996; Rhodes et al., 1996) was produced from a mouse immunized with a GST fusion protein containing the entire 367 amino acid rat $\mathrm{Kv} \beta 2$ polypeptide. K17/70 subsequently was found to bind to a synthetic peptide corresponding to amino acids 1-17 of $\mathrm{Kv} \beta 2$. Hybridomas were grown in $\mathrm{BALB} / \mathrm{c}$ mice for production of ascites fluid, as previously described (Trimmer et al., 1985). Immuno- globulins were purified by ammonium sulfate precipitation, followed by DEAE chromatography, as described (Trimmer et al., 1985).

Electrophysiology. For conduction studies the sural branch of the sciatic nerve was desheathed and dissociated into single fibers, as discussed above, to maximize drug access to all nerve fibers. Dissected nerves were sealed with Vaseline in a chamber to two pairs of platinum wire electrodes for stimulation and recording (Vabnick et al., 1997). The temperature was measured with a small thermistor in the chamber and held at either RT or at $37^{\circ} \mathrm{C}$. The nerve was immersed in a constantly stirred and perfused oxygenated Locke's solution containing $5 \mathrm{~mm}$ D-glucose, to which pharmacological agents were added as desired. The stimulus was adjusted to $\sim 10 \%$ above the level that elicited a maximum response. The compound action potential obtained from excitation was amplified, digitized, recorded, and analyzed on a laboratory computer.

\section{RESULTS}

\section{Normal distribution of Kv1.1}

The normal Kv1.1 distribution in teased nerve fibers is shown in Figure $1 a$. Immunoreactivity was strongest in the region in which the diameter of the axon increased in comparison to that of the nodal diameter; this region is called the juxtaparanode (Rosenbluth, 1984). This juxtaparanodal localization of Kv1.1 channels is identical to that demonstrated by Mi et al. (1995). The intensity of Kv1.1 staining was usually stronger near the paranode and decreased toward the internode (Fig. 1a). The cytoplasmic perinuclear region of the Schwann cell also had Kv1.1 immunoreactivity (data not shown). Figure $1 b$ shows the same nodal region as in Fig. 1a, labeled for myelin-associated glycoprotein (MAG). MAG immunofluorescence is found primarily in the terminal loops, paranodal regions, and the periaxonal space of mature myelinating Schwann cells (Martini and Schachner, 1986). MAG was used as a myelin marker to aid in identifying the nodal region and integrity of the myelin. Figure $1, c$ and $d$, shows doublelabeling for $\mathrm{Kv} 1.1 \mathrm{~K}^{+}$channels and $\mathrm{Na}^{+}$channels, respectively. Close comparison reveals clear gaps between the nodal $\mathrm{Na}^{+}$ channel staining and the juxtaparanodal Kv1.1 immunoreactivity, regions corresponding to the paranodes. Figure $1, c$ and $d$, has 

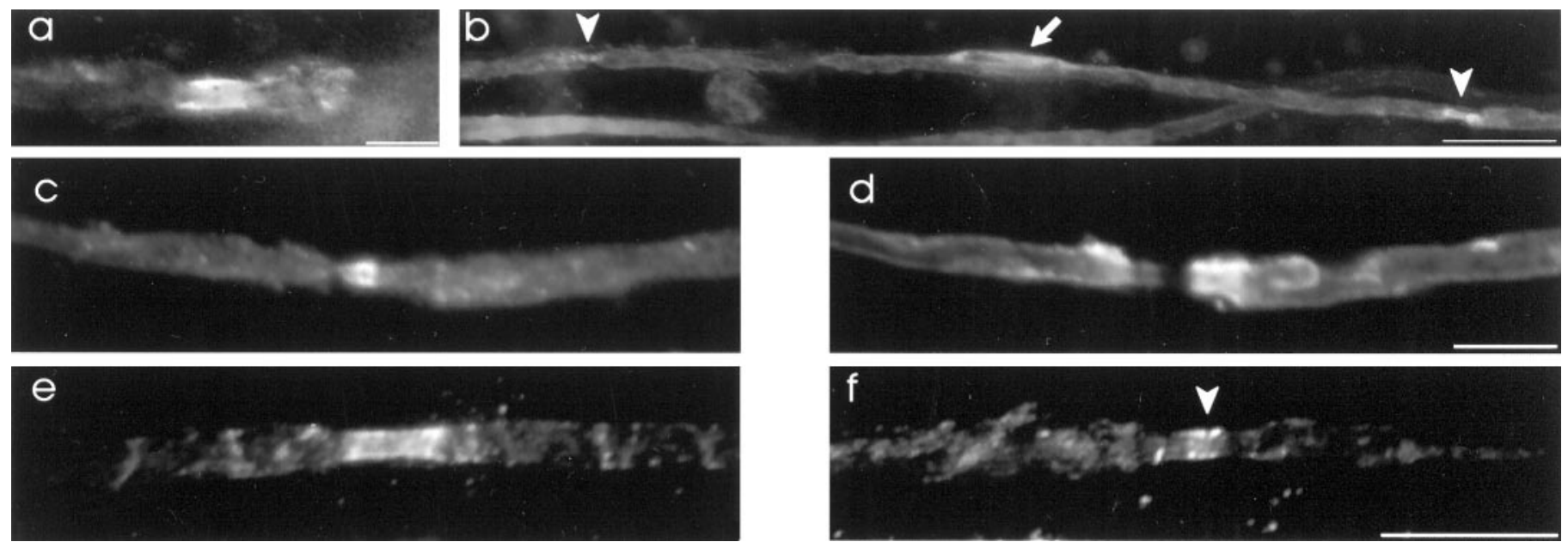

Figure 3. Kv1.1 is found at the node of Ranvier during remyelination. $a$, A node, at 14 dpi, labeled for Kv1.1. $b$ (composite), A full internode, at 14 dpi, with Kv1.1 immunoreactivity at nodes (arrowheads) and in the perinuclear region of the Schwann cell (arrow). c, $d$, A node double-labeled for Kv1.1 (c) and MAG $(d) . e, f$, Double-labeling for Kv1.1 $(e)$ and $\mathrm{Na}^{+}$channels $(f)$ indicates that the two distributions overlap at the node $($ arrowhead $)$ but that Kv1.1 staining extends beyond the $\mathrm{Na}^{+}$channel immunoreactivity. Scale bars: $a, c, d, 12 \mu \mathrm{m} ; b, e, f, 25 \mu \mathrm{m}$.

been merged in Figure 1e to show the gaps (arrowheads) between Kv1.1 and $\mathrm{Na}^{+}$channel immunoreactivity.

Teased nerve fibers were advantageous because they allowed individual axons to be followed for significant distances to verify remyelination. Because this preparation requires some mechanical manipulation, experiments were repeated on cryosectioned nerves from perfused animals. The nerve cryosections were double-labeled to determine the normal distributions of Kv1.1 and MAG, and the results were compared with those from teased fibers. Figure $1 f$ shows a cryosectioned node of Ranvier labeled for Kv1.1 immunoreactivity. The juxtaparanodes are labeled, and there is a gradual decrease in the intensity toward the internode. Figure $1 g$ shows the paranodal regions of this node labeled for MAG. In all essential aspects the Kv1.1 labeling patterns in cryosectioned nerves appear to be identical to those of teased axon preparations.

The specificity of the rabbit polyclonal Kv1.1 antibody was determined by preincubating the antibody with its peptide antigen and then by double-labeling control nerve fibers with the blocked Kv1.1 antibody and anti-MAG. Figure $1, h$ and $i$, shows the results. The preabsorbed anti-Kv1.1 in Figure $1 h$ did not label the juxtaparanodes or any other part of the node. Perinuclear cytoplasmic regions of Schwann cells were also devoid of any staining (data not shown). The MAG immunoreactivity appeared normal and unchanged, as seen in Figure $1 i$. These findings are interpreted to mean that anti-Kv1.1 was specific for the potassium channel Kv1.1 and correctly defined the normal distribution. Furthermore, a similar localization pattern was seen with both monoclonal and polyclonal anti-Kv1.1 antibodies.

\section{Kv1.1 in demyelinated axons}

By 6 dpi of lysolecithin, most disrupted myelin had been cleared by phagocytosis, and broad regions of demyelinated axons were seen with spherical cells, primarily myelin-laden macrophages, attached (Hall and Gregson, 1971). Demyelinated nerve fibers were double-labeled for both $\mathrm{Na}^{+}$channel and Kv1.1 immunofluorescence. $\mathrm{Na}^{+}$channel clusters at nodes of Ranvier appeared to be preserved, and by identifying these sites within demyelinated regions, it was possible to evaluate the stability of Kv1.1 domains in the axolemma. Figure 2 is representative of the results at this stage. Figure $2 a$ shows the Kv1.1 distribution in a demyelinated zone. The corresponding $\mathrm{Na}^{+}$channel cluster is seen in Figure $2 b$. Kv1.1 immunofluorescence appears to have lost the precise localization seen in normal nerve fibers and has moved into paranodal and nodal zones. The decreased intensity at the site occupied by $\mathrm{Na}^{+}$channels (Fig. $2 a$, arrowhead) suggests some residual exclusion of Kv1.1 at the node. Figure 2, $c$ and $d$, shows another node in a demyelinated region at $6 \mathrm{dpi}$, but in this instance Kv1.1 immunoreactivity is below detectable levels. To determine the Kv1.1 distribution immediately after demyelination, we counted and characterized former nodes (identified as $\mathrm{Na}^{+}$channel clusters) in demyelinated regions as having no Kv1.1 staining (Fig. 2c), diff use graded Kv1.1 that extended through the node (Fig. 2a), or paranodal Kv1.1 that did not overlap with the nodal $\mathrm{Na}^{+}$channel immunofluorescence (data not shown). At 6 dpi, $49 \%$ of nodal regions had diff use graded Kv1.1 staining, $40 \%$ had no Kv1.1 staining, and $11 \%$ had paranodal Kv1.1 immunofluorescence $(n=37)$. One day later $(7 \mathrm{dpi})$ the relative number of nodal regions without any Kv1.1 staining increased to $79 \%$, whereas that with diff use Kv1.1 staining decreased to $21 \%(n=$ 24). Neither paranodal nor juxtaparanodal Kv1.1 was observed at any nodes from demyelinated regions at this stage. Heminodes, sites in which one side of the node was demyelinated and the other unaffected, had preserved $\mathrm{Na}^{+}$channel clusters at the node and Kv1.1 clusters in the juxtaparanode on the myelinated side but no Kv1.1 immunoreactivity in the demyelinated zone (data not shown). These results suggest that clusters of $\mathrm{Kv} 1.1 \mathrm{~K}^{+}$ channels are more labile in the axolemma than are $\mathrm{Na}^{+}$channel clusters and that local signals from associated Schwann cells are necessary to preserve their aggregation.

\section{Kv1.1 at early stages of remyelination}

At 7 dpi neither demyelinated axons nor the few Schwann cells associated with these axons were MAG-positive, but in some cases diff use low-level Kv1.1 staining was detected in fully demyelinated regions of the axon. In cases in which a cell was clearly associated with the axon, presumably a Schwann cell just beginning the process of remyelination, Kv1.1 labeling often appeared. Figure $2 e$ shows a demyelinated axon with an associated cell $(*)$. Kv1.1 staining can be seen on either side of the cell with a graded 

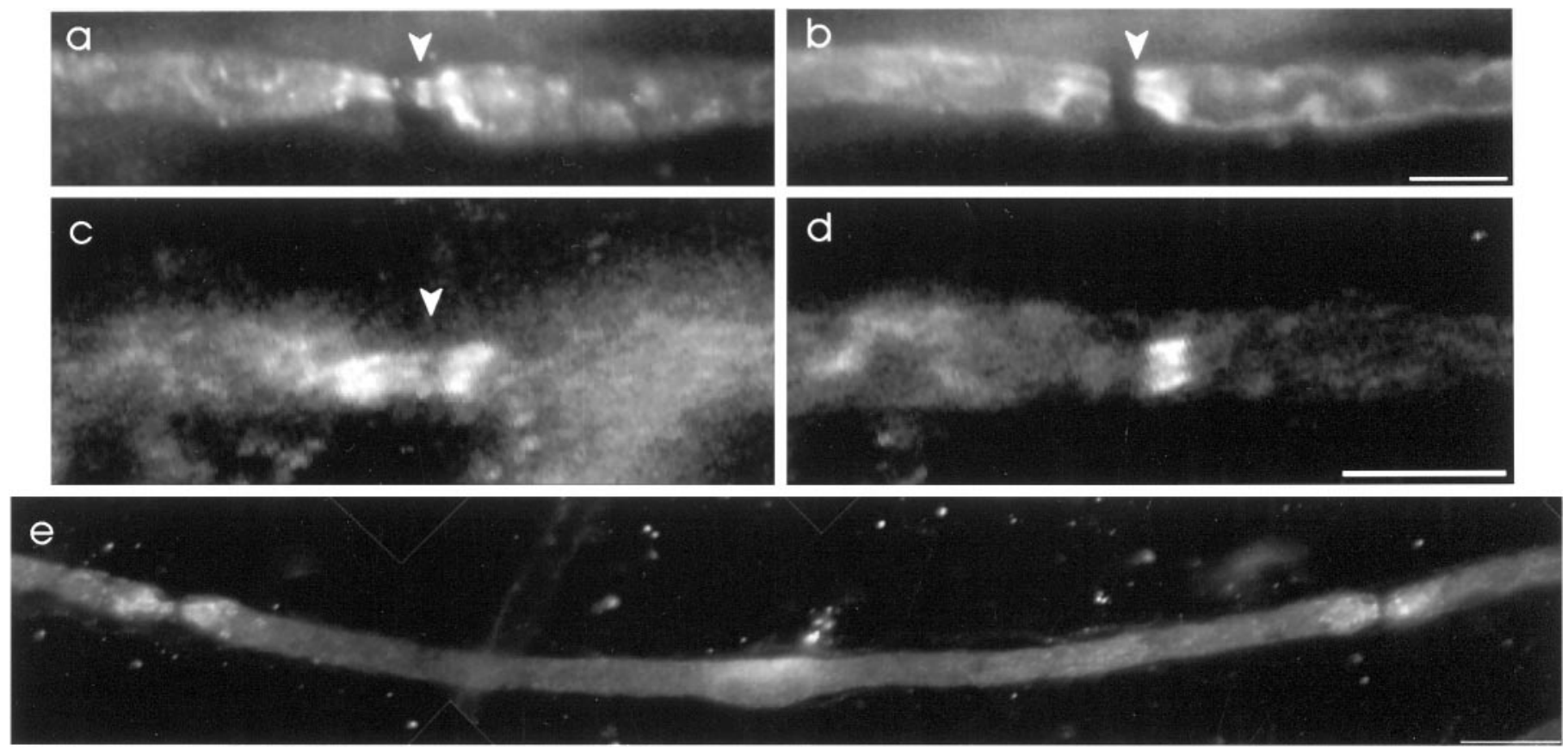

Figure 4. Kv1.1 channels are clustered at the paranodes by $24 \mathrm{~d}$ after lysolecithin injection. $a, b$, A node, at 24 dpi, double-labeled for Kv1.1 ( $a$ ) and MAG $(b)$ indicates that paranodal Kv1.1 and MAG overlap (arrowheads). $c, d$, A node, at 24 dpi, double-labeled for Kv1.1 (c) and Na ${ }^{+}$channels $(d)$. Kv1.1 is aggregated toward the paranodes, leaving a gap (arrowhead) that overlaps with $\mathrm{Na}^{+}$channel immunoreactivity. $e$ (composite), A full internode, at $32 \mathrm{dpi}$, has paranodal/juxtaparanodal Kv1.1 immunofluorescence. Further, the perinuclear region of the Schwann cell has Kv1.1 staining. Scale bars: $a, b, 12 \mu \mathrm{m} ; c, d, 25 \mu \mathrm{m} ; e, 30 \mu \mathrm{m}$.

intensity that increases closer to the cell. It was not possible to determine whether this label represents channels in the axolemma or a population of Kv1.1 channels in elongating cytoplasmic Schwann cell processes. However, because staining of the perinuclear cytoplasm was not observed, a feature that is pronounced in normal myelinating Schwann cells and actively remyelinating Schwann cells, the immunolabel may be axonal. Figure $2 f$ shows that the same region has no MAG immunoreactivity.

Figure 2, $g$ and $h$, shows an example of remyelination at $9 \mathrm{dpi}$. At this stage, Schwann cells had associated with axons and had begun to extend processes. These Schwann cells also had diffuse Kv1.1 staining (Fig. 2g). However, the paranodes, juxtaparanodes, and the region between Schwann cell processes, the presumptive node (arrowhead), were conspicuously Kv1.1-negative. Along these processes there was evidence for MAG expression (Fig. $2 h$ ), indicating that active remyelination had begun. It has been shown that MAG is first expressed after one and a half turns of Schwann cell processes (Martini and Schachner, 1986).

At 10 dpi the average internodal length (defined here as the distance between the middle of consecutive nodal gaps) in remyelinating zones was $147 \pm 48 \mu \mathrm{m}$. At this early stage of repair no presumptive nodal regions, of 22 sites examined, had Kv1.1 immunoreactivity. In contrast to the absence of Kv1.1 staining, it has been shown previously that $\mathrm{Na}^{+}$channels cluster at presumptive nodes by 10 dpi (Dugandzija-Novakovic et al., 1995).

\section{Kv1.1 is present at the node during remyelination}

Clear axonal Kv1.1 staining was seen first in remyelinating axons at $12 \mathrm{~d}$ after lysolecithin injection. Surprisingly, however, at this stage the Kv1.1 $\mathrm{K}^{+}$channels were found in the node. The number of fibers with this distribution increased during the next several days until it became the dominant staining pattern. At 12 dpi new nodes of Ranvier were counted and characterized as having either nodal Kv1.1 or no label. Nodes were included only if a full internode, with its adjacent nodes, was well preserved and could be followed for its entire length. This criterion allowed for a determination of the state of remyelination, i.e., whether the fiber was intact and undisrupted or remyelinated. Fibers $\sim 4 \mu \mathrm{m}$ in diameter and larger were considered remyelinated if the measured internodal distance was $<350 \mu \mathrm{m}$ (Hildebrand et al., 1985). Further, MAG staining on newly remyelinated nerve fibers appeared more uniform across the surface of the myelin than in fibers that did not undergo demyelination and subsequent remyelination (data not shown). These criteria allowed for identification of remyelinated nerve fibers. Transition nodes, sites in which one side of the node was undisrupted but the other side was demyelinated and then subsequently remyelinated, were not included. Of the 42 nodes that were included in the count, $8 \%$ had nodal Kv1.1 staining, whereas the remaining 92\% had no label. The average internodal length was $126 \pm 62 \mu \mathrm{m}$. It appeared that all myelinating Schwann cells were both MAG-positive and had Kv1.1 immunoreactivity in perinuclear regions.

By 13 dpi the number of presumptive nodes with Kv1.1 immunoreactivity increased to $12 \%$, and the remaining $88 \%$ of nodes were without any detectable $\operatorname{Kv1.1}(n=42)$. The average internodal length was $159 \pm 66 \mu \mathrm{m}$. The large uncertainty in internodal length reflects the fact that the extent of remyelination may be highly variable.

Figure $3 a$ shows an example of this intense nodal Kv1.1 staining at a new node of Ranvier at 14 dpi. The Kv1.1 immunoreactivity fills the region between the two Schwann cells and appears to be in the axolemma. Figure $3 b$ shows a full internode with a node at each end (arrowheads). The right node has nodal Kv1.1 staining, but the left is unlabeled. Further, the perinuclear region of the Schwann cell has pronounced Kv1.1 immunoreactivity (arrow). 


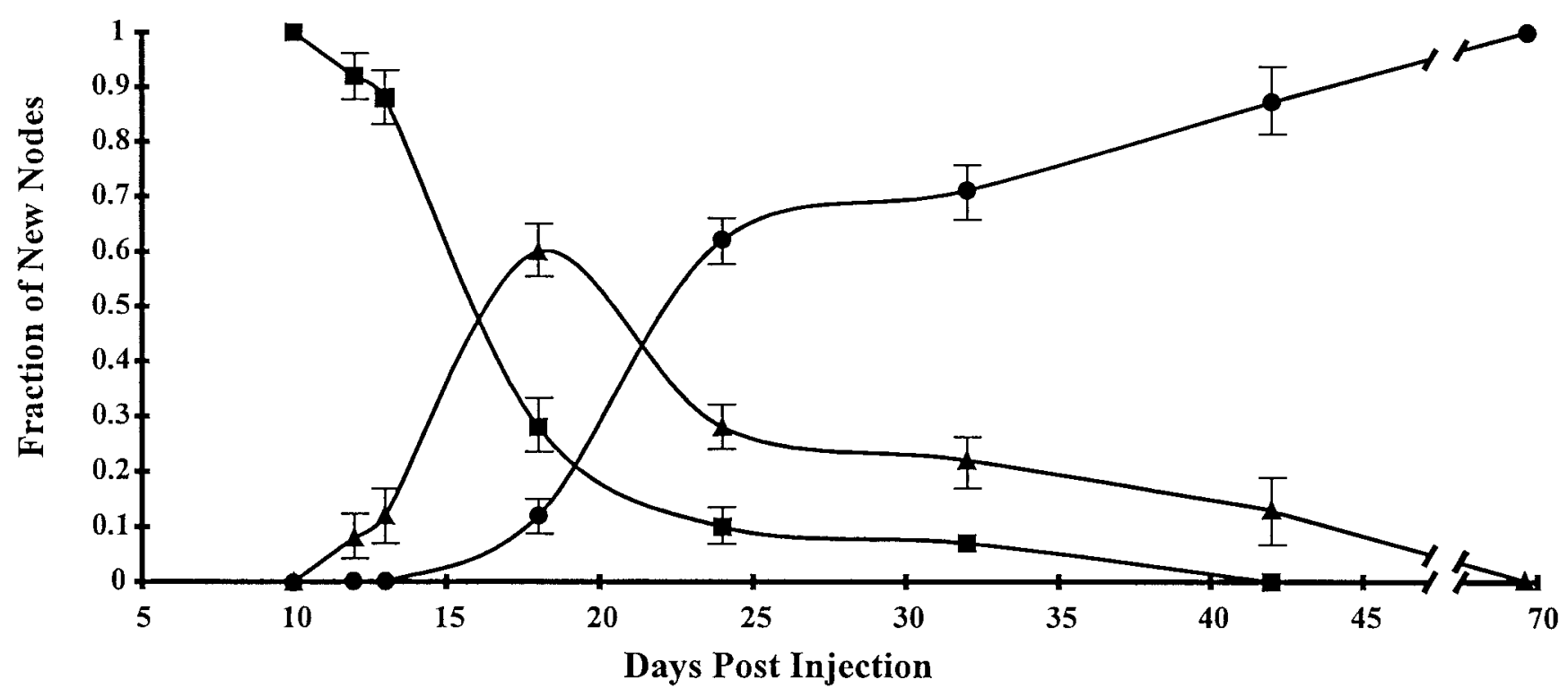

Figure 5. Kv1.1 channel distributions during remyelination plotted as the fraction of new nodes observed versus days postinjection of lysolecithin. $\mathbf{\square}$, No nodal, paranodal, or juxtaparanodal labeling; $\mathbf{\Lambda}$, nodal immunoreactivity only; $\boldsymbol{\bullet}$, paranodal labeling (with a gap at the node). Error bars indicate \pm SEM.

At 18 dpi three distinct nodal labeling patterns for Kv1.1 were present in remyelinated nerve fibers, in addition to the perinuclear staining of Schwann cells observed at all times later than 9 dpi. These three patterns included the following: (1) a new distribution that was paranodal with a striking gap in Kv1.1 labeling directly at the node of Ranvier (see below for description and figure), (2) no nodal or paranodal Kv1.1 staining (as in Fig. $2 g$ ), and (3) immunoreactivity at nodes only (as in Fig. 3a). Although all three of these patterns were present, only $12 \%$ had paranodal clustering of Kv1.1, 60\% of nodes were labeled uniformly, and $28 \%$ of nodes had no Kv1.1 labeling $(n=86)$. At this time the average internodal length was $223 \pm 84 \mu \mathrm{m}$. Figure $3 c$ is representative of the majority of new nodes at this time and shows focal nodal Kv1.1 staining. When the Kv1.1 staining is compared with the MAG labeling of the same site, as shown in Figure $3 d$, it is possible to see that the Kv1.1 extends between the two Schwann cells and occupies the entire gap between areas of MAG immunofluorescence. This gap corresponds to the new node and is very narrow, only $\sim 4 \mu \mathrm{m}$ in width. Figure $3 e$ shows a broad region of Kv1.1 immunofluorescence distributed between two apposing Schwann cells. Figure $3 f$ shows that a $\mathrm{Na}^{+}$channel cluster (arrowhead) also is well defined at this time and can be found colocalized with the $\mathrm{K}^{+}$channels at the node. However, it is clear from a comparison between the two channel types that the Kv1.1 staining extends beyond the focal $\mathrm{Na}^{+}$channel cluster in a much broader distribution.

\section{Kv1.1 clusters at paranodes}

At $24 \mathrm{dpi}$, the percentage of new nodes with nodal label and of those with no label decreased. The number of nerve fibers with new paranodal Kv1.1 staining increased so that this became the dominant pattern. Figure $4 a$ is representative of this paranodal distribution. Paranodal staining extended to the edge of the juxtaparanodal region and in a few cases into the juxtaparanode, where the diameter of the axon increases (see Fig. 1, $a$ or $c$, for comparison). The most striking feature of this labeling pattern was the gap in Kv1.1 staining found directly at the node of Ranvier, where $\mathrm{Na}^{+}$channels were clustered in high density.
MAG staining, shown in Figure $4 b$, was continuous along the surface of the myelin but was most intense at the paranodes. It is important to note that the edges of MAG staining always coincided with the edges of the paranodal Kv1.1 staining (arrowheads in Fig. $4 a, b$, right paranode), suggesting some involvement of the Schwann cell in clustering. Figure $4 c$, from another experiment, shows that the gap in Kv1.1 staining (arrowhead) colocalizes with the nodal $\mathrm{Na}^{+}$channel cluster seen in Figure $4 d$. Further, because this gap is very small $(\sim 2 \mu \mathrm{m})$, this particular example may represent very early paranodal clustering. The average internodal length of remyelinated nerve fibers at $24 \mathrm{dpi}$ was $261 \pm 54 \mu \mathrm{m}$. At this stage $62 \%$ of nodal regions had paranodal Kv1.1 labeling, $28 \%$ had nodal staining, and $10 \%$ of nodes had no Kv1.1 immunofluorescence $(n=115)$.

To determine whether the Kv1.1 potassium channel distribution in remyelinated nerve fibers continued to change until it became exclusively juxtaparanodal again or, if it remained near the node, in paranodal regions, we determined Kv1.1 distributions at 32, 42, and 69 dpi. Figure $4 e$ shows a full internode from a 32 dpi rat. Kv1.1 labeling is present in two locations: the perinuclear region of the Schwann cell and the paranodes/juxtaparanodes. A gap is present at both nodes, and both nodes have similar Kv1.1 clustering. Despite the fact that these nodes had the same general appearance, consecutive nodes did not always have the same Kv1.1 distributions during early remyelination. The average internodal length at $32 \mathrm{dpi}$ in remyelinated rat axons was $256 \pm 49 \mu \mathrm{m}$. In $71 \%$ of new nodes Kv1.1 immunofluorescence was found in paranodal regions, $22 \%$ had uniform nodal label, and only $7 \%$ of nodes had no Kv1.1 staining $(n=72)$. By 42 dpi $87 \%$ of nodal regions had paranodal distributions of Kv1.1, $13 \%$ had nodal Kv1.1, and nodal regions devoid of Kv1.1 immunoreactivity were not seen $(n=31)$. At 42 dpi Kv1.1 appeared in paranodal and into juxtaparanodal regions. However, when juxtaparanodal Kv1.1 staining was seen, it encroached into the paranode rather than into the internode. At $69 \mathrm{dpi}$, all nodal regions $(n=31)$ from remyelinated axons had paranodal/juxtaparanodal 

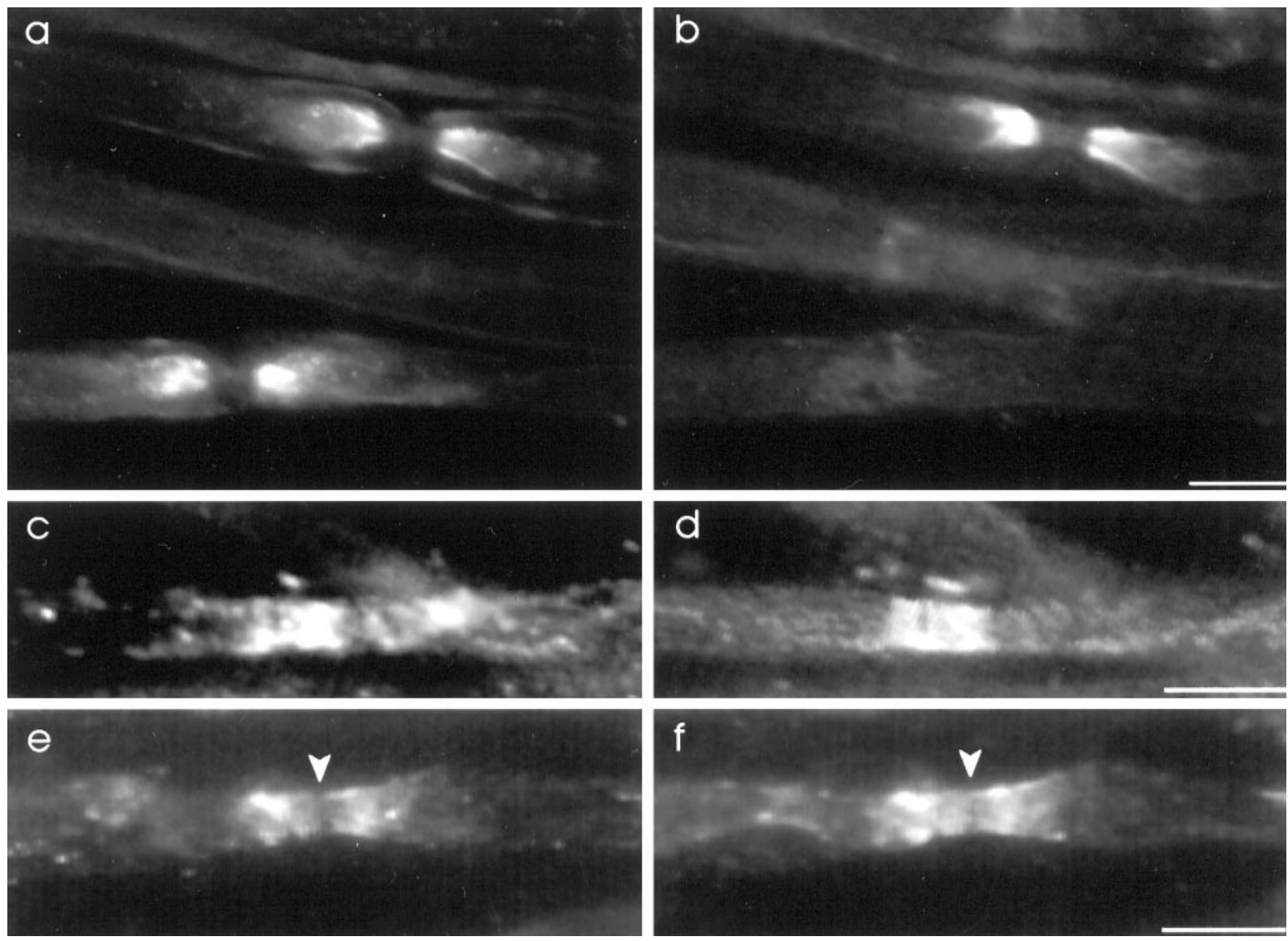

Figure 6. Kv1.1 and Kv1.2 are colocalized in most normal and remyelinated nerve fibers. $a, b$, Two normal nodes double-labeled for Kv1.1 ( $a$ ) and Kv1.2 (b). Both nodes of Ranvier have juxtaparanodal Kv1.1, but only the top one has juxtaparanodal Kv1.2. $c$, d, At 19 dpi, double-labeling for Kv1.1 (c) and Kv1.2 $(d)$ indicates that during remyelination both channel types may be present at newly forming nodes. $e, f$, A node, at 24 dpi, with an early gap (arrowheads) in both Kv1.1 (e) and Kv1.2 (f) immunoreactivity. Scale bars: $a, b, 25 \mu \mathrm{m} ; c-f, 12 \mu \mathrm{m}$.

$\mathrm{K}^{+}$channels and were seen in distributions similar to those at 32 and 42 dpi.

\section{Nodal Kv1.1 is transient}

The data indicate that Kv1.1 is initially absent from the nodal region, transiently expressed at the node of Ranvier, and finally clustered at the paranodes/juxtaparanodes. The different types of Kv1.1 staining throughout the remyelination process and how these patterns relate to each other are shown in Figure 5; the figure is a summary of the quantitative data discussed above. As the Kv1.1 distributions were counted, the internodal lengths were measured also. The average internodal length after remyelination reached a maximum at $\sim 270 \mu \mathrm{m}$, in good agreement with previously reported values after remyelination (Hildebrand et al., 1985; Dugandzija-Novakovic et al., 1995). One interesting result of these measurements was that the greatest changes in internodal length and architecture of the new myelin coincided with the time (13 through $24 \mathrm{dpi}$ ) when Kv1.1 distributions were most variable and when both $\mathrm{Na}^{+}$and $\mathrm{K}^{+}$channels colocalized at the node.

\section{Kv1.2 colocalizes with Kv1.1 in most axons}

Within the Shaker Kv1.x family of $\mathrm{K}^{+}$channels, different subunits may come together to form heteromultimers with functional properties intermediate between those of homomultimeric $\mathrm{K}^{+}$channels (Isacoff et al., 1990; Ruppersberg et al., 1990; Salkoff et al., 1992). In addition to Kv1.1, Kv1.2 is present in axons of the peripheral nervous system. Figure $6 a$ shows two nodes, with juxtaparanodes labeled for
Kv1.1. However, in Figure $6 b$, the same region labeled for Kv1.2, only one of these axons has Kv1.2 immunoreactivity. In preparations double-labeled for both Kv1.1 and Kv1.2, we found that $85 \%$ of juxtaparanodes had both Kv1.1 and Kv1.2 staining that precisely colocalized. However, in $15 \%$ of nerve fibers, only Kv1.1 immunoreactivity was seen (total number of nodes counted $=198$ ). Within a given axon the pattern of $\mathrm{K}^{+}$channel expression was constant. Thus, consecutive nodal regions in the same fiber either had both Kv1.1 and Kv1.2 immunoreactivity or had only Kv1.1 staining. Nodal regions with only Kv1.2 were not seen.

The possibility that differences in $\mathrm{K}^{+}$channel expression in neurons reflected populations of either motor or sensory nerve fibers was investigated by double-labeling dorsal and ventral spinal roots with antibodies against both Kv1.1 and Kv1.2. Dorsal and ventral root preparations each had fibers with both Kv1.1 and Kv1.2 immunolabeling and fibers with only Kv1.1 staining.

\section{Kv1.1 and Kv1.2 colocalize throughout remyelination}

During remyelination Kv1.1 is present at the node of Ranvier and then finally clusters at the paranodes. Double-labeling experiments were performed on nerve fibers during remyelination to determine whether Kv1.2 colocalizes with Kv1.1 throughout this process. Figure $6, c$ and $d$, is representative of the distributions observed at $19 \mathrm{dpi}$ and shows nodal colocalization, indicating that both Kv1.1 (Fig. $6 c$ ) and Kv1.2 (Fig. $6 d$ ) appear in the node of Ranvier during remyelination. Figure $6, e$ and $f$, shows early 
No Drug

+ TEA

Control

RT

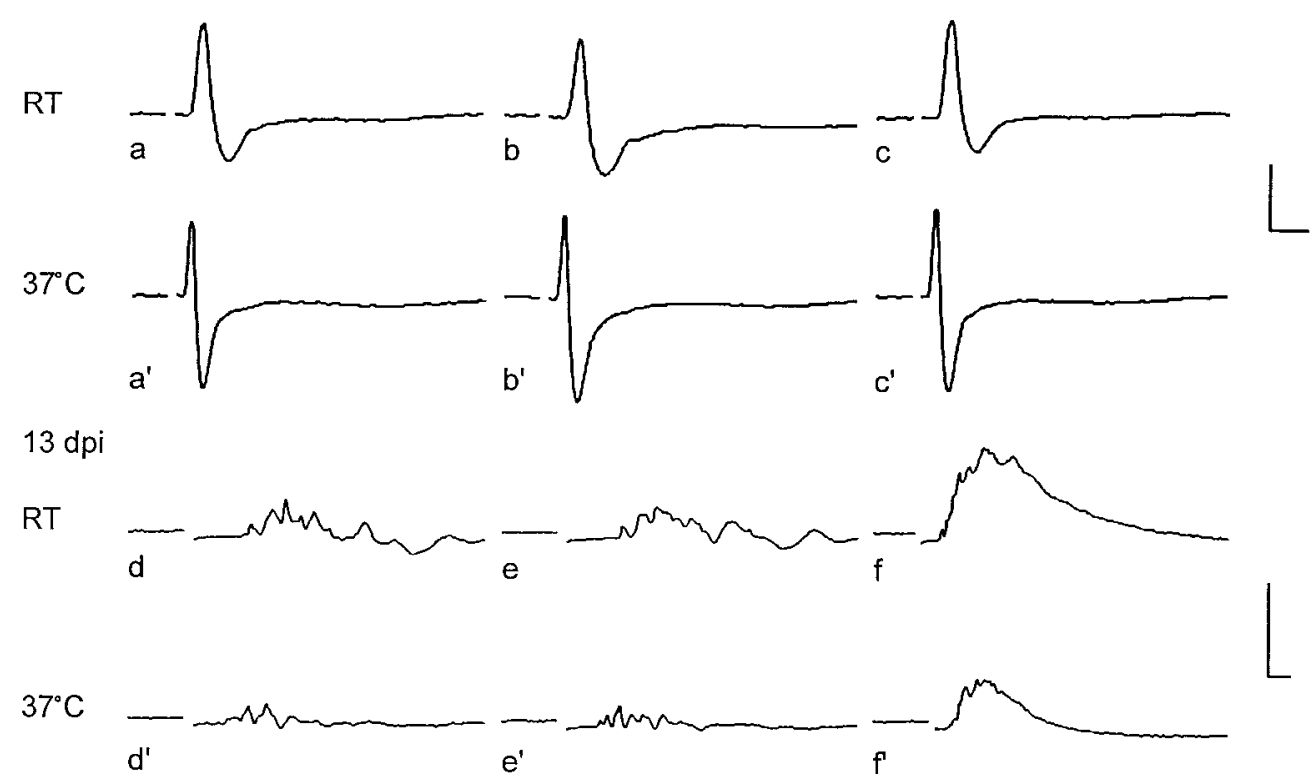

Figure 7. Compound action potentials (CAPs) in control and remyelinating 13 dpi nerves. CAPs were measured both at room temperature $(R T)$ and at $37^{\circ} \mathrm{C}$, as indicated at the left of each row. At each temperature the traces are shown before the addition of drug (left) and in $10 \mathrm{~mm}$ tetraethylammonium (TEA; center) or $1 \mathrm{mM}$ 4-aminopyridine (4-AP; right). Calibration: (Control), $0.25 \mathrm{mV}, 1 \mathrm{msec}$; (13 dpi), $0.2 \mathrm{mV}, 1 \mathrm{msec}$ in traces $d, d^{\prime}, e$, and $e^{\prime}$ and 2 msec in $f$ and $f^{\prime}$.

paranodal clustering of Kv1.1 and Kv1.2, respectively. The arrowhead defines the nodal gap at which $\mathrm{Na}^{+}$channels are presumed to be clustered. At both 19 and 24 dpi all new nodes that were immunoreactive for Kv1.1 also stained positively for Kv1.2. However, this may be attributable to the inherent difficulty of finding clearly identifiable new nodes in remyelinated nerve fibers at this stage. We cannot be certain that nodes with just one channel subtype are absent during early remyelination. Indeed, at $69 \mathrm{dpi}$, examples were found of remyelinated nerve fibers with only Kv1.1 immunofluorescence at paranodal/juxtaparanodal regions in consecutive nodes (data not shown).

\section{$\mathrm{K}^{+}$channel blockers and conduction properties}

The $\mathrm{K}^{+}$channel blockers tetraethylammonium $\left(\mathrm{TEA}^{+}\right)$and 4-aminopyridine (4-AP) were used to assess $\mathrm{K}^{+}$channel sensitivity during and after remyelination. The drug $\mathrm{TEA}^{+}\left(K_{\mathrm{D}}\right.$ for mouse Kv1.1 = $0.3 \mathrm{~mm}$ and for rat Kv1.2 = 560 mM) (Grissmer et al., 1994) blocks $\mathrm{K}^{+}$channels from the external side of the pore region when applied extracellularly (MacKinnon and Yellen, 1990), and 4-AP ( $K_{\mathrm{D}}$ for mouse Kv1.1 $=290 \mu \mathrm{M}$ and for rat $\mathrm{Kv1.2}=590 \mu \mathrm{M})\left(\right.$ Grissmer et al., 1994) blocks $\mathrm{K}^{+}$channels by diff using across the cell membrane and acting from inside the cell, possibly near the binding site for the Shaker inactivation peptide (Castle et al., 1994; Stephens et al., 1994). Compound action potentials (CAPs) were recorded before demyelination and at 13, 19, 27, and $34 \mathrm{~d}$ after lysolecithin injection. In Figure 7, traces $a$ and $a^{\prime}$ show representative CAPs from a normal sciatic nerve at both $\mathrm{RT}$ and at $37^{\circ} \mathrm{C}$. The CAP duration was reduced and conduction velocity increased at $37^{\circ} \mathrm{C}$, but there was little further effect. Normal axons were insensitive to both $10 \mathrm{~mm} \mathrm{TEA}^{+}$ (traces $b$ and $b^{\prime}$ ) and $1 \mathrm{~mm}$ 4-AP (traces $c$ and $c^{\prime}$ ). At $13 \mathrm{~d}$ after lysolecithin injection most axons were at an early stage of remyelination. Consequently, the CAP was small and reflected the presence of a heterogeneous population of fibers varying in the extent of remyelination and, therefore, in conduction delays (trace $d$ ). Conduction was very temperature-sensitive, a characteristic of demyelinated axons (trace $\left.d^{\prime}\right)$. TEA ${ }^{+}(10 \mathrm{~mm})$ had no effect on conduction properties (traces $e$ and $e^{\prime}$ ). However, on the addition of $1 \mathrm{~mm} 4-\mathrm{AP}$, the shape of the CAP changed significantly (traces $f$ and $f^{\prime}$ ), increasing in both the amplitude and the duration of the CAP. The CAP remained temperature-sensitive (trace $f^{\prime}$ ). Figure 8 shows results for nerves at 19, 27, and $34 \mathrm{dpi}$. At 19 dpi for both $\mathrm{RT}$ and $37^{\circ} \mathrm{C}$, there was very little $\mathrm{TEA}^{+}$ sensitivity, but temperature changes resulted in significant alteration of the CAP (traces $a, a^{\prime}, b$, and $b^{\prime}$ ). On the addition of 4-AP the duration of the CAP changed dramatically (traces $c$ and $c^{\prime}$ ); the time scale for trace $c$ in Figure 8 is 12 times that for CAPs in the absence of any drug. Increased temperature sensitivity persisted (trace $c^{\prime}$ ). At 27 dpi the nerve remained temperaturesensitive and TEA ${ }^{+}$-insensitive (traces $d, d^{\prime}, e$, and $e^{\prime}$ ). With the addition of 4-AP (traces $f$ and $f^{\prime}$ ), the amplitude of the negative phase decreased. However, the duration of the CAP was not increased significantly. By $34 \mathrm{dpi}$, the CAP closely resembled that of normal nerves both in the presence and absence of TEA ${ }^{+}$ (traces $g, g^{\prime}, h$, and $h^{\prime}$ ). However, on the addition of 4-AP only minor deviations from the control trace appeared, suggesting that, at this late stage of remyelination, few axons remained 4-AP (trace $i$ ) and temperature-sensitive (trace $i^{\prime}$ ). These records show that remyelinated axons are very sensitive to 4-AP precisely at the time when the Kv1.1 $\mathrm{K}^{+}$channels have been shown to be present at the node of Ranvier.

\section{Mitomycin-C delays Kv1.1 clustering at the node}

The clustering of Kv1.1 appeared to be related to the degree of remyelination. To test for an essential role of Schwann cells in the redistribution of Kv1.1 channels, we added the mitotic inhibitor mitomycin-C to the lysolecithin injection. The drug blocks proliferation of Schwann cells and delays remyelination (Hall and Gregson, 1974). At 18 dpi, remyelinating Schwann cells were not present, Kv1.1 staining was not detected at heminodes (data not shown), and focal clusters of Kv1.1 immunoreactivity could not be found in demyelinated zones. Figure $9 a$ shows a heminode at 24 dpi (arrowhead). The demyelinated region to the right of the heminode has increased Kv1.1 staining in the axolemma but no 
Figure 8. Compound action potentials in remyelinating nerves at 19,27 , and 34 dpi. Temperature and drug conditions are as indicated and as in Figure 7. Calibration: (19 dpi) $0.05 \mathrm{mV}, 1 \mathrm{msec}$ in $a$, $a^{\prime}, b$, and $b^{\prime}, 12 \mathrm{msec}$ in $c$, and $3 \mathrm{msec}$ in $c^{\prime} ;(27$ dpi ), $0.5 \mathrm{mV}, 1 \mathrm{msec}$; (34 dpi ), 0.5 $\mathrm{mV}, 1 \mathrm{msec}$ in $g, g^{\prime}, h, h^{\prime}$, and $i^{\prime}$ and 2 msec in $i$.

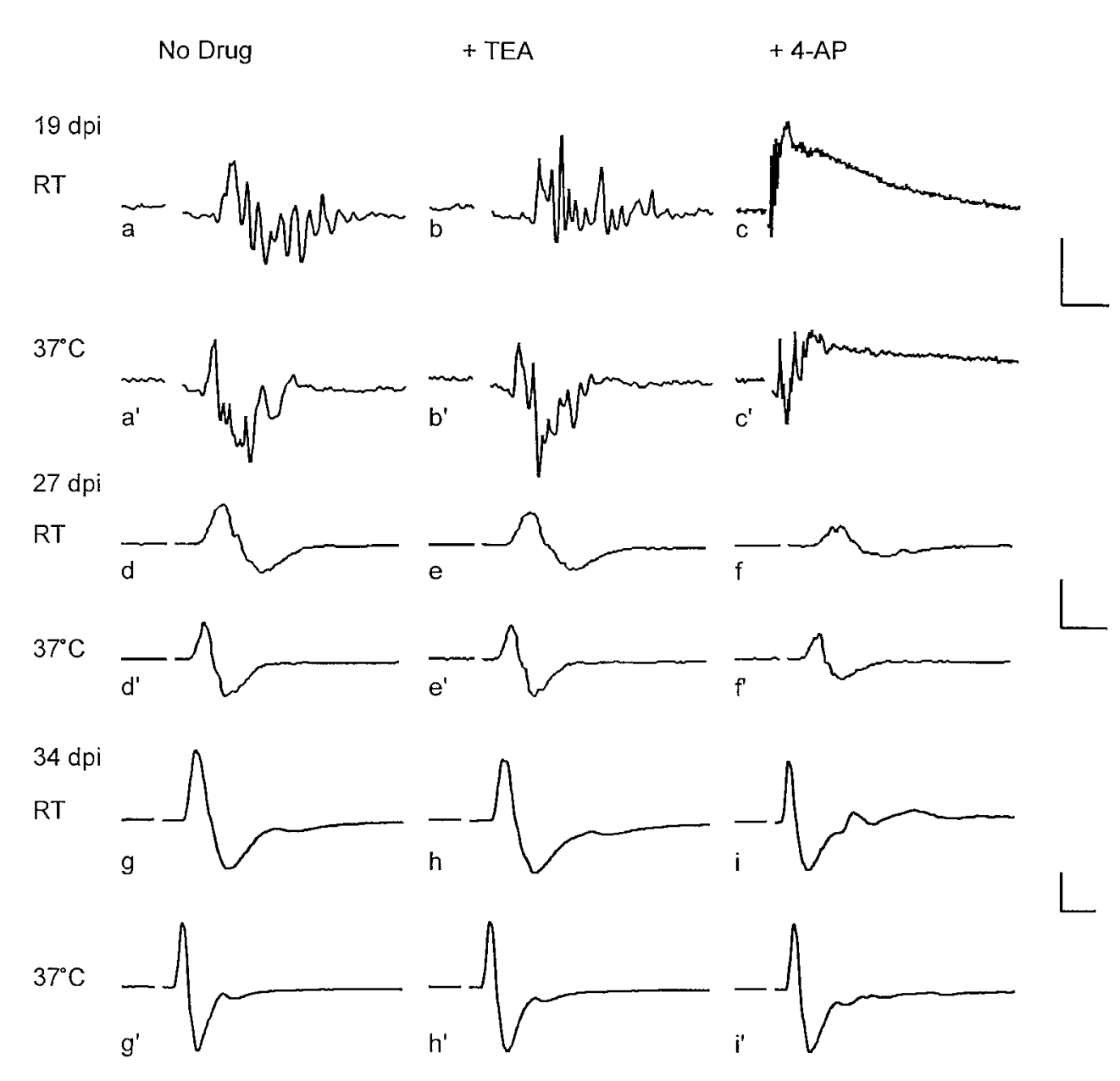

MAG immunoreactivity (MAG not shown). In the absence of Schwann cell association it appears that Kv1.1 is present in the axolemma, but no precise subcellular localization of these channels occurs. Eventually, new nodes form, presumably as mitomycin-C effects dissipate, and $\mathrm{K}^{+}$channels appear in the nodal gap. This pattern is present at much longer times than normal, and even as late as $47 \mathrm{~d}$ after lysolecithin and mitomycin-C injection many examples of nodal Kv1.1 staining were found, suggesting that remyelination was delayed. Comparison with Figure 5 indicates that nodal $\mathrm{K}^{+}$channel staining ought to be very rare by this time. Figure $9 b$ shows nodal Kv1.1 staining (arrowhead) from a 47 dpi mitomycin-C-injected animal. Figure $9 c$ shows the corresponding MAG immunofluorescence, with a gap between apposing Schwann cells.

\section{$\mathrm{Kv1.1}$ and $\mathrm{Kv} \boldsymbol{\beta} 2$ colocalize during remyelination}

In addition to the membrane-spanning $\alpha$-subunits, cytoplasmic $\beta$-subunits may be found in association with $\mathrm{K}^{+}$channels. In particular, the $\mathrm{Kv} \beta 2$ subunit has been suggested to mediate efficient cell surface expression of $\mathrm{K}^{+}$channels (Shi et al., 1996). To investigate the possibility that $\mathrm{Kv} \beta 2$ participates in Kv1.1 expression before and during remyelination in the peripheral nervous system, we double-labeled nerve fibers with antibodies against $\mathrm{Kv1.1}$ and $\mathrm{Kv} \beta 2$. Figure 9, $d$ and $e$, shows that Kv1.1 (Fig. 9d) was found to colocalize with the cytoplasmic $\mathrm{Kv} \beta 2$ subunit (Fig. 9e). During remyelination, at $19 \mathrm{dpi}, \mathrm{Kv} \beta 2$ was present in the node of Ranvier. Figure $9 f$ shows that Kv1.1 immunoreactivity overlapped precisely with the Kv $\beta 2$ immunofluorescence shown in Figure $9 g$.
Furthermore, $\operatorname{Kv} \beta 2$ immunofluorescence was not detected in the perinuclear region of Schwann cells (data not shown).

\section{DISCUSSION}

\section{Normal axons}

The first voltage-clamp experiments on nodes of Ranvier, conducted on amphibian axons, demonstrated the presence of voltage-dependent $\mathrm{Na}^{+}$and $\mathrm{K}^{+}$channels (Dodge and Frankenhaeuser, 1958; Frankenhaeuser, 1962). The latter were sensitive to block by TEA and were shown to be important in repolarizing the nodal membrane after action potential conduction (Hille, 1967). In contrast, Chiu and Ritchie (1980) later showed that voltage-dependent $\mathrm{K}^{+}$channels are absent from mammalian nodes of Ranvier. In experiments on rabbit myelinated axons, they measured large $\mathrm{K}^{+}$currents only after acute treatment to loosen the myelin from the axonal membrane. This observation suggested that voltage-dependent $\mathrm{K}^{+}$channels are present only in paranodal and internodal regions. Further, these authors argued that a large $\mathrm{K}^{+}$leakage current in mammalian nodes was sufficient for rapid repolarization (Chiu et al., 1979). More recently, immunocytochemical methods have allowed for a more precise localization of voltage-dependent $\mathrm{K}^{+}$channels, demonstrating Kv1.1 and Kv1.2 clustered in juxtaparanodal regions, probably as heteromultimeric channels (Wang et al., 1993; Mi et al., 1995).

Using immunofluorescence, we have confirmed the localization of Kv1.1 and Kv1.2 in juxtaparanodes of normal myelinated rat 

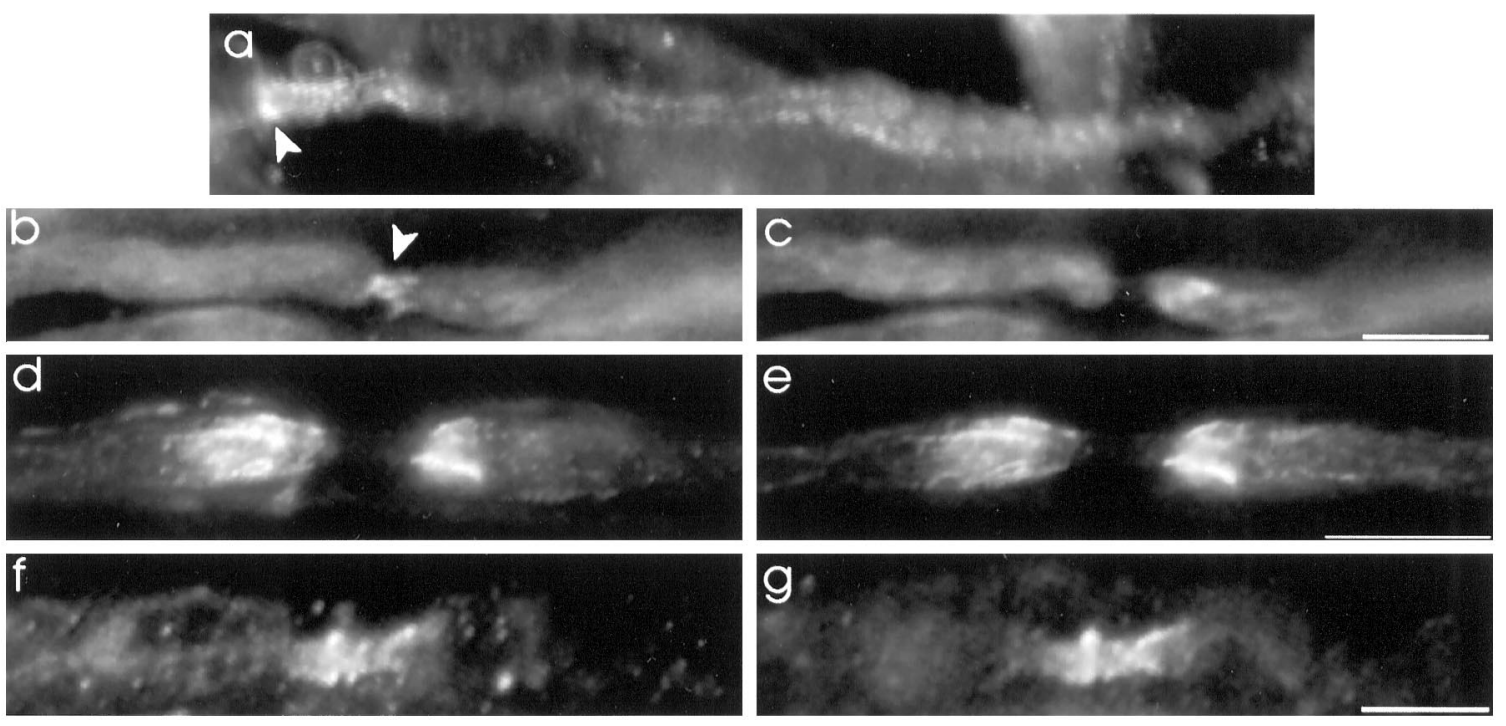

Figure 9. Mitomycin-C delays remyelination and $\mathrm{K}^{+}$channel clustering, and the $\mathrm{K}^{+}$channel subunit $\mathrm{Kv} \beta 2$ is colocalized with $\mathrm{Kv} 1.1$ in normal and remyelinating nerve fibers. $a$, A demyelinated axon, at $24 \mathrm{dpi}$ of mitomycin-C and lysolecithin, labeled for Kv1.1. A heminode in $a$ (arrowhead) with the demyelinated zone to the right has diffuse labeling of Kv1.1 in the absence of Schwann cell interaction. $b, c$, A node, at 47 dpi with mitomycin-C and lysolecithin, illustrates Kv1.1 immunoreactivity within the nodal gap. $d, e$, A normal node double-labeled for Kv1.1 (d) and Kv $\beta 2(e) . f, g$, A node, at 19 dpi, double-labeled for $\operatorname{Kv} 1.1(f)$ and $\operatorname{Kv} \beta 2(g)$, showing that $\operatorname{Kv} \beta 2$ is colocalized with $\operatorname{Kv} 1.1$ at the node during remyelination. Scale bars: $a$, $d$, $e$, $25 \mu$ m; $b, c, f, g, 12 \mu \mathrm{m}$.

nerve fibers. Further, we found that in most cases these channels colocalized, but in $15 \%$ of the nodes examined only Kv1.1 was present. Because consecutive nodes within individual axons had the same labeling pattern, it appears that differing patterns of expression between axons are controlled by neurons rather than by Schwann cells. The functional significance of these heterogeneous distributions is unknown but does not appear to represent differences between motor and sensory neurons, because both dorsal and ventral spinal roots had Kv1.1/Kv1.2 coexpression or only Kv1.1 staining.

Double-labeling experiments with anti- $\mathrm{Na}^{+}$channel and Kv1.1 channel antibodies have revealed that their respective subcellular domains do not overlap but instead are very distinct and are separated by a small gap. Using immunoelectron microscopy, Mi et al. (1995) have shown that Kv1.1 is localized at juxtaparanodes, and Novakovic et al. (1996) have demonstrated that clusters of $\mathrm{Na}^{+}$channels in normal adult fibers could be detected only at the node between adjacent Schwann cells. The gap seen in our double-labeling experiments corresponds to the paranode, a region in which the terminal loops of Schwann cells form tight axoglial junctions and in which the caliber of the axon is narrowed in comparison with the internodal diameter (Rosenbluth, 1984). The fact that neither Kv1.1 nor $\mathrm{Na}^{+}$channels could be found in paranodal regions suggests that both channel types may be excluded from the specialized sites in which the Schwann cell paranodal loops come in close contact with the axon.

\section{Demyelinated fibers}

Seven days postinjection of lysolecithin represents the time when many internodes are stripped entirely of myelin, and Schwann cells have not yet begun remyelination. At this time Kv1.1 clusters could not be detected at the majority of former juxtaparanodal regions. In the remaining cases $(21 \%)$ demyelinated zones had diffuse Kv1.1 immunoreactivity that extended through the paranode and node. By 9 dpi, the earliest stage of remyelination, Kv1.1 clusters were undetectable. These results suggest that aggregation of these channels, in the absence of any Schwann cell interaction, is more labile than that of $\mathrm{Na}^{+}$channel clusters. The latter retained their precise location for periods of at least 1 week, although by 2 weeks $\mathrm{Na}^{+}$channel clusters were not seen if Schwann cell proliferation was prevented (Dugandzija-Novakovic et al., 1995). Wiley-Livingston and Ellisman (1982) showed that during remyelination rows of intramembranous particles appeared and were associated with terminal loops of myelin during the formation of new nodes of Ranvier. Some of these particles may represent $\mathrm{K}^{+}$channels. Wang et al. (1995) have examined Kv1.1 distributions in the hypomyelinating mouse strain Trembler. They reported altered Kv1.1 distributions, with channels no longer confined to juxtaparanodal regions and more diff use than in wild-type mice. Most importantly, the largest changes in $\mathrm{K}^{+}$ channel redistribution were seen in hypomyelinated regions. These observations are consistent with the hypothesis that Kv1.1 clustering requires Schwann cell interaction for maintenance and stabilization in specific subcellular domains.

Inhibition of Schwann cell proliferation by mitomycin-C delayed $\mathrm{K}^{+}$channel redistribution and clustering. At 24 dpi, demyelinated axons with broad zones of Kv1.1 immunoreactivity were seen, whereas in the absence of this drug these channels were already restricted mainly to paranodal/juxtaparanodal zones at this time. Neuronal proteins may be essential for clustering channels (Kim et al., 1995), but their action alone appears to be insufficient to direct aggregates to specific regions. Likewise, Vabnick et al. (1996, 1997) have shown that Schwann cells are necessary for $\mathrm{Na}^{+}$channel clustering during development and that long-term maintenance of these clusters also requires Schwann cell interaction. It is interesting to note that during remyelination $\mathrm{Na}^{+}$channel clusters first appear in two separate bands, which then fuse to form one aggregate at the node (Dugandzija-Novakovic et al., 1995). In contrast, $\mathrm{K}^{+}$channels are first detected at the node and then split and cluster to paranodal regions. In each case, aggregation seems to be directed by adher- 


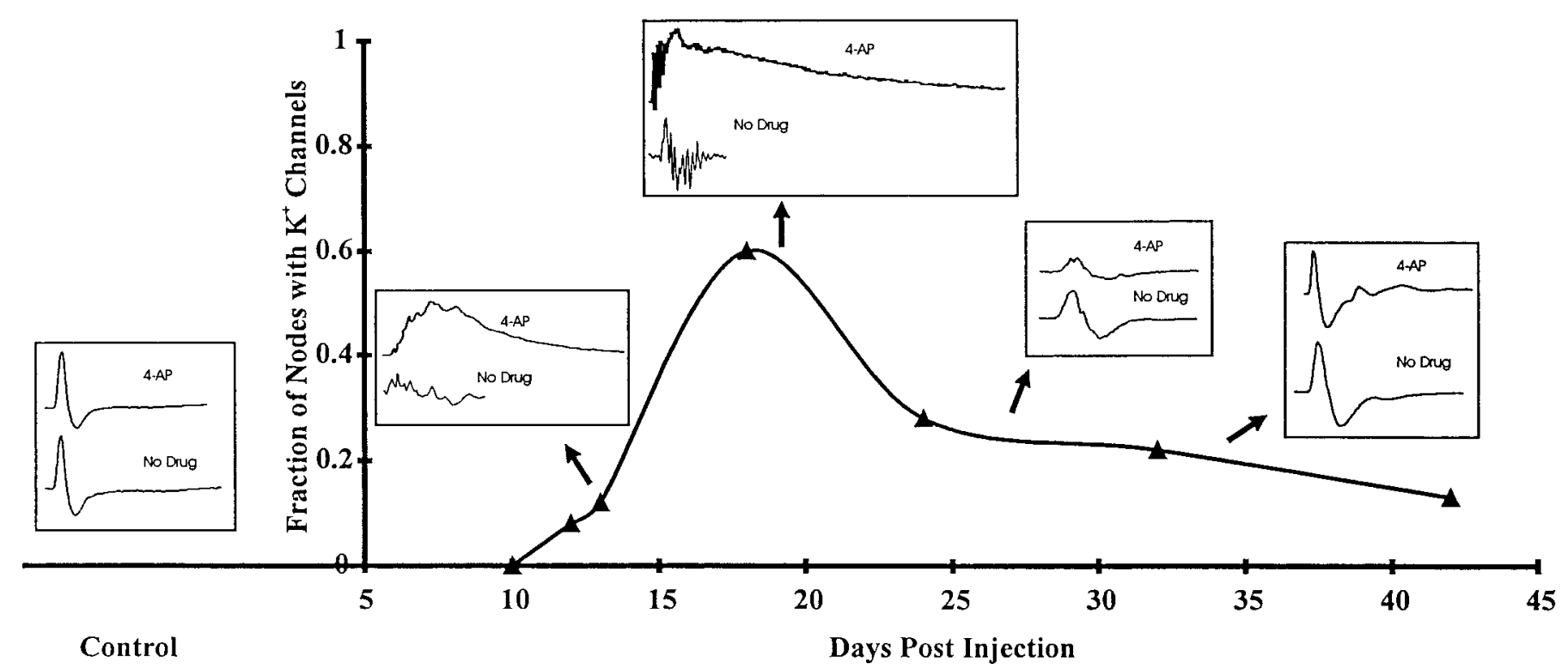

Figure 10. Compound action potential amplitude and duration depend on the fraction of nodes with $\mathrm{K}^{+}$channels $(\boldsymbol{\Delta})$ and the consequent sensitivity to 4-aminopyridine $(4-A P)$.

ent Schwann cells that have become committed to myelination. However, the clustering of these two channel types occurs in opposite directions and at different times, suggesting unique mechanisms.

Shi et al. (1996) have shown that Kv1.2 and Kv $\beta 2$ immunoreactivity colocalizes in regions adjacent to nodes of Ranvier in rat cerebellar cortex. Further, they showed that in transfected mammalian cells $\operatorname{Kv} \beta 2$ associates with $\mathrm{Kv} 1.2 \alpha$-subunits and acts as a chaperone to promote $\mathrm{N}$-linked glycosylation, increased stability, and more efficient cell surface expression of Kv1.2. Because Kv $\beta 2$ was found to be colocalized with $\mathrm{K}^{+}$channels at the node during remyelination, it may have a similar function in the peripheral nervous system. Recently, Rhodes et al. (1997) have shown colocalization of $\mathrm{Kv1.1}, \mathrm{Kv} 1.2$, and $\mathrm{Kv} \beta 2$ at paranodal regions in the CNS. The fact that paranodal regions in axons from both the peripheral and CNS have similar staining patterns suggests that these regions have similar requirements for $\mathrm{K}^{+}$channel expression and function.

We have used the $\mathrm{K}^{+}$channel blocking drugs TEA ${ }^{+}$and 4-AP to investigate the physiological function of Kv1.1 and Kv1.2 under both normal and pathological conditions. The drug TEA ${ }^{+}$, when applied extracellularly, acts by occluding the $\mathrm{K}^{+}$channel pore (Hille, 1967; MacKinnon and Yellen, 1990). In contrast, 4-AP has been shown to block mouse Kv1.1 channels by diff using across the membrane and acting in close proximity to the binding site for the inactivation peptide (Stephens et al., 1994). The mechanism for action of $\mathrm{TEA}^{+}$allows for an investigation of $\mathrm{K}^{+}$channel accessibility during remyelination, whereas 4-AP circumvents the need for accessibility and allows for assessment of $\mathrm{K}^{+}$channel function in the normal and demyelinated nerve fiber. Bostock et al. (1981) have studied the effects of $4-\mathrm{AP}$ and $\mathrm{TEA}^{+}$on both normal rat spinal roots and spinal roots that were demyelinated, using diphtheria toxin. They observed very little or no effect of $20 \mathrm{~mm}$ $\mathrm{TEA}^{+}$and $5 \mathrm{~mm}$ 4-AP on CAPs from normal roots. We have similar results for normal sciatic nerve, suggesting that Kv1.1 and $\mathrm{Kv} 1.2 \mathrm{~K}^{+}$channels do not contribute to the repolarization of the axon under normal conditions. Chiu and Ritchie (1981) have speculated that juxtaparanodal $\mathrm{K}^{+}$channels may play an impor- tant part in stabilizing action potential discharge at the node, i.e., preventing reexcitation of the nodal membrane. The observation that myelinated, regenerating axons treated with 4-AP elicit bursting activity after a single stimulus is consistent with this idea (Kocsis et al., 1982).

By combining electrophysiology and immunofluorescence, we can evaluate the $\mathrm{K}^{+}$channel distribution and its effect on function during remyelination. The sensitivity to 4-AP appears to be dependent both on the number of nerve fibers with Kv1.1 and Kv1.2 and on the distribution of these $\mathrm{K}^{+}$channels. Figure 10 shows that, as remyelination progressed through 19 dpi, the effect of $1 \mathrm{mM}$ 4-AP became more dramatic: both the amplitude and duration of the CAP increased. Furthermore, there is a correlation between the number of nodal regions with $\mathrm{K}^{+}$channels and the effect of 4-AP. The presence of $\mathrm{K}^{+}$channels at the node may result in decreased conduction efficiency. The sensitivity to 4-AP decreased by 27 and 34 dpi as remyelination continued. Because 4-AP is permeant and is likely to reach these channels, this observation suggests that Kv1.1 and Kv1.2 contribute less to conduction during later stages of remyelination because they are sequestered increasingly under the myelin in paranodal regions. Improved tightness of axoglial junctions may be responsible for the decreased sensitivity to 4-AP at later stages of remyelination $\left(27+\right.$ dpi). Thus, after demyelination, $\mathrm{K}^{+}$channels do not alter conduction properties solely by exposure as paranodes are disrupted. Indeed, their strongest influence in reducing excitability during remyelination occurs during their transient presence at the node.

\section{REFERENCES}

Bekele-Arcuri Z, Matos MF, Manganas MM, Strassle BW, Monaghan MM, Rhodes KJ, Trimmer JS (1996) Generation and characterization of subtype-specific monoclonal antibodies to $\mathrm{K}^{+}$channel $\alpha$ - and $\beta$-subunit polypeptides. Neuropharmacology 35:851-865.

Bostock H, Sears TA (1978) The internodal axon membrane: electrical excitability and continuous conduction in segmental demyelination. J Physiol (Lond) 280:273-301.

Bostock H, Sears TA, Sherratt RM (1981) The effects of 4-aminopyridine and tetraethylammonium ions on normal and demyelinated mammalian nerve fibres. J Physiol (Lond) 313:301-315. 
Castle NA, Fadous SR, Logothetis DE, Wang GK (1994) 4-Aminopyridine binding and slow inactivation are mutually exclusive in rat Kv1.1 and Shaker potassium channels. Mol Pharmacol 46:1175-1181.

Chiu SY, Ritchie JM (1980) Potassium channels in nodal and internodal axonal membrane of mammalian myelinated fibres. Nature 284:170-171.

Chiu SY, Ritchie JM (1981) Evidence for the presence of potassium channels in the paranodal region of acutely demyelinated mammalian single nerve fibres. J Physiol (Lond) 313:415-437.

Chiu SY, Ritchie JM, Rogart RB, Stagg D (1979) A quantitative description of membrane currents in rabbit myelinated nerve. J Physiol (Lond) 292:149-166.

Dodge F, Frankenhaeuser B (1958) Membrane currents in isolated frog nerve fibre under voltage clamp conditions. J Physiol (Lond) 143:76-90.

Dugandzija-Novakovic S, Koszowski AG, Levinson SR, Shrager P (1995) Clustering of $\mathrm{Na}+$ channels and node of Ranvier formation in remyelinating axons. J Neurosci 15:492-502.

Frankenhaeuser B (1962) Delayed currents in myelinated nerve fibres of Xenopus laevis investigated with voltage clamp technique. J Physiol (Lond) 160:40-45.

Grissmer S, Nguyen AN, Aiyar J, Hanson DC, Mather RJ, Gutman GA, Karmilowicz MJ, Auperin DD, Chandy KG (1994) Pharmacological characterization of five cloned voltage-gated $\mathrm{K}^{+}$channels, types Kv1.1, $1.2,1.3,1.5$, and 3.1 , stably expressed in mammalian cell lines. Mol Pharmacol 45:1227-1234.

Hall SM, Gregson NA (1971) The in vivo and ultrastructural effects of injection of lysophosphatidyl choline into myelinated peripheral nerve fibres of the adult mouse. J Cell Sci 9:769-789.

Hall SM, Gregson NA (1974) The effects of mitomycin C on remyelination in the peripheral nervous system. Nature 252:303-305.

Hildebrand C, Kocsis JD, Berglund S, Waxman SG (1985) Myelin sheath remodelling in regenerated rat sciatic nerve. Brain Res 358:163-170.

Hille B (1967) The selective inhibition of delayed potassium currents in nerve by tetraethylammonium ion. J Gen Physiol 50:1287-1302.

Isacoff EY, Jan YN, Jan LY (1990) Evidence for the formation of heteromultimeric potassium channels in Xenopus oocytes. Nature 345:530-534.

Kim E, Niethammer M, Rothschild A, Jan YN, Sheng M (1995) Clustering of Shaker type $\mathrm{K}^{+}$channels by interaction with a family of membrane-associated guanylate kinases. Nature 378:85-88.

Kocsis JD, Waxman SG, Hildebrand C, Ruiz JA (1982) Regenerating mammalian nerve fibres: changes in action potential waveform and firing characteristics following blockage of potassium conductance. Proc R Soc Lond [Biol] 217:77-87.

MacKinnon R, Yellen G (1990) Mutations affecting TEA blockade and ion permeation in voltage-activated $\mathrm{K}^{+}$channels. Science 250:276-279.

Martini R, Schachner M (1986) Immunoelectron microscopic localization of neural cell adhesion molecules (L1, N-CAM, and MAG) and their shared carbohydrate epitope and myelin basic protein in developing sciatic nerve. J Cell Biol 103[Pt 1]:2439-2448.

Mi H, Deerinck TJ, Ellisman MH, Schwarz TL (1995) Differential distribution of closely related potassium channels in rat Schwann cells. J Neurosci 15:3761-3774.

Novakovic SD, Deerinck TJ, Levinson SR, Shrager P, Ellisman MH (1996) Clusters of axonal $\mathrm{Na}^{+}$channels adjacent to remyelinating Schwann cells. J Neurocytol 25:403-412.
Poltorak M, Sadoul R, Keilhauer G, Landa C, Fahrig T, Schachner M (1987) Myelin-associated glycoprotein, a member of the L2/HNK-1 family of neural cell adhesion molecules, is involved in neuronoligodendrocyte and oligodendrocyte-oligodendrocyte interaction. J Cell Biol 105:1893-1899.

Rasband MN, Schwarz TL, Ellisman MH, Schachner M, Novakovic SD, Shrager P (1996) Shaker-type potassium channel (Kv1.1) clustering in remyelinating rat axons. Soc Neurosci Abstr 22:50.

Rhodes KJ, Barrezeuta NX, Monaghan MM, Bekele-Arcuri Z, Nakahira K, Nawoschik S, Schechter LE, Trimmer JS (1996) Voltage-gated K ${ }^{+}$ channel $\beta$ subunit polypeptides: expression and distribution of $\operatorname{Kv} \beta 1$ and $\operatorname{Kv} \beta 2$ in adult rat brain. J Neurosci 16:4846-4860.

Ritchie JM (1982) Sodium and potassium channels in regenerating and developing mammalian myelinated nerves. Proc R Soc Lond [Biol] 215:273-287.

Ritchie JM, Rang HP, Pellegrino R (1981) Sodium and potassium channels in demyelinated and remyelinated mammalian nerve. Nature 294:257-259.

Rosenbluth J (1984) Membrane specialization at the nodes of Ranvier and paranodal and juxtaparanodal regions of myelinated central and peripheral nerve fibers. In: The node of Ranvier (Zagoren JC, Fedoroff S, eds), pp 31-65. Orlando, FL: Academic.

Ruppersberg JP, Schroter KH, Sakmann B, Stocker M, Sewing S, Pongs O (1990) Heteromultimeric channels formed by rat brain potassiumchannel proteins. Nature 345:535-537.

Salkoff L, Baker K, Butler A, Covarrubias M, Pak MD, Wei A (1992) An essential "set" of $\mathrm{K}^{+}$channels conserved in flies, mice, and humans. Trends Neurosci 15:161-166.

Shi G, Nakahira K, Hammond S, Rhodes KJ, Schechter LE, Trimmer JS (1996) Beta subunits promote $\mathrm{K}^{+}$channel surface expression through effects early in biosynthesis. Neuron 16:843-852.

Shrager P (1987) The distribution of sodium and potassium channels in single demyelinated axons of the frog. J Physiol (Lond) 392:587-602.

Shrager P (1989) Sodium channels in single demyelinated mammalian axons. Brain Res 483:149-154.

Stephens GJ, Garratt JC, Robertson B, Owen DG (1994) On the mechanism of 4-aminopyridine action on the cloned mouse brain potassium channel mKv1.1. J Physiol (Lond) 477[Pt 2]:187-196.

Trimmer JS, Trowbridge IS, Vacquier VD (1985) Monoclonal antibody to a membrane glycoprotein inhibits acrosome reaction and associated $\mathrm{Ca}^{2+}$ and $\mathrm{H}^{+}$fluxes of sea urchin sperm. Cell 40:697-703.

Vabnick I, Novakovic SD, Levinson SR, Schachner M, Shrager P (1996) The clustering of axonal sodium channels during development of the peripheral nervous system. J Neurosci 16:4914-4922.

Vabnick I, Novakovic SD, Messing A, Chiu SY, Levinson SR, Schachner M, Shrager P (1997) Sodium channel distribution in axons of hypomyelinated and MAG null mutant mice. J Neurosci Res 50:321-336.

Wang H, Kunkel DD, Martin TM, Schwartzkroin PA, Tempel BL (1993) Heteromultimeric $\mathrm{K}^{+}$channels in terminal and juxtaparanodal regions of neurons. Nature 365:75-79.

Wang H, Allen ML, Grigg JJ, Noebels JL, Tempel BL (1995) Hypomyelination alters $\mathrm{K}^{+}$channel expression in mouse mutants shiverer and Trembler. Neuron 15:1337-1347.

Wiley-Livingston CA, Ellisman MH (1982) Return of axonal and glial membrane specializations during remyelination after telluriuminduced demyelination. J Neurocytol 11:65-80. 